\title{
Theory of Fiber Optic Co- and Counter-pumped Raman Polarizers
}

\author{
Stefan Wabnitz, Victor V. Kozlov ${ }^{1,2}$, Javier Nuño ${ }^{3}$, Juan Diego Ania-Castañón ${ }^{3}$ \\ 1. University of Brescia, Department of Information Engineering, Via Branze 38, 25123, Brescia, Italy \\ 2. St.-Petersburg University, Department of Physics, St.-Petersburg, Petrodvoretz, 198504, Russia \\ 3. Instituto de Optica, Consejo Superior de Investigaciones Cientificas (CSIC), 28006 Madrid, Spain
}

Fiber optic Raman amplifiers are polarization-dependent by their nature: the state of polarization (SOP) of the outcoming signal beam strongly depends on the SOP of the pump beam. Thus, in isotropic fibers the SOP of the signal acquires the SOP of the pump beam towards the output, because the Raman gain for a signal SOP that is aligned along the pump SOP is two orders of magnitude larger than the Raman gain for the orthogonal signal polarization. However, telecommunication fibers are not isotropic but exhibit a birefringence which stochastically changes along the propagation distance. Moreover, the pump and the signal beams experience a different birefringence. This mismatch has the consequence that SOPs of the two beams do not change in unison when they propagate through the fiber. On average, the signal beam "sees" the two orthogonal polarizations of the pump beam with equal frequence. This situation is characteristic to high-PMD fibers (here, PMD stands for the polarization mode dispersion), namely, with PMD coefficients $>0.05 \mathrm{ps} / \mathrm{km}^{1 / 2}$. In this case the signal beam experiences an average Raman gain which is half of the maximal Raman gain. Thus a simple scalar model of Raman amplification is appropriate for describing high-PMD fibers, since all polarization-dependent properties of the Raman gain are washed out by fiber birefringence.

The situation is radically different when using low-PMD fibers. These fibers stand close to isotropic fibers with respect to the polarization dependence of the Raman gain. Hence, one may hope to restore the repolarization properties of ideal fiber optic Raman amplifiers owing to the intrinsic polarization dependence of Raman gain. Indeed, as experimentally demonstrated in a recent paper by Martinelli et.al. in Ref. [1], in addition to the function of amplification of light, Raman amplifiers based on low-PMD fibers are also able to convert a set of polarization scrambled signal beams into a set of fully polarized beams with their SOPs tightly aligned along the pump SOP. Here, by scrambled beams we mean a set of fully polarized beams, whose SOPs are uniformly (or randomly) distributed over the Poincare sphere. In this case the Raman amplifier turns into a Raman polarizer, a device that along with the amplification of unpolarized beams simultaneously converts them into fully polarized beams.

The goal of our work is to construct a theory which is capable of predicting the main characteristics of Raman polarizers: their overall gain, the average SOP of the outcoming signal beam, and the output signal degree of polarization (DOP) that results whenever the polarizer is fed by unpolarized light. The final result of our theory is the formulation of a set of partial differential equations governing the evolution of the Stokes parameters of the signal and the pump beams with space-dependent coefficients, which in its turn can be found by solving a simple set of ordinary differential equations. When numerically implemented, this procedure takes a computational time of the order of one minute on a laptop computer. This represents a substantial advance over standard Monte-Carlo numerical procedures for the numerical modelling of light propagation in randomly birefringent fibers, which typically require solving the propagation equations for each of $\sim 10000$ realizations of fibers, followed by a proper statistical averaging over the ensemble of all these realizations. Given that a single propagation run takes about one second, we may estimate overall computational time of three hours. This is the time which is needed to process only a single input SOP: for processing scrambled beams consisting of 150 SOPs randomly distributed over the Poincare sphere, the calculation time would then rise up to 17 days.

By taking advantage of the accurate and fast computations that are enabled by our theory, we performed fullscale studies of the performances of Raman polarizers in both co-propagating as well as counter-propagating geometries. In particular, we found that the output signal DOP is different for different SOPs of the pump beam: in other words, some polarizers perform better than others. We also found that the average gain of a Raman polarizer is significantly higher than the gain of a typical (i.e., based on a relatively high-PMD fiber) Raman amplifier operating under similar conditions. Such gain enhancement is obtained thanks to the efficient alignment of the signal SOP along the pump SOP, that is along the maximally amplified polarization mode. We also found that, when using low-PMD fibers, the PMD coefficient is not always the appropriate quantity to characterise the properties of a Raman polarizer. Indeed, one should rather use a pair of quantities - the fiber beat length and its correlation length. Based on our theory, we extensively characterized the performance of Raman polarizers for wide range of fibers with different beat and correlation lengths [2].

\section{References}

[1] M. Martinelli, M. Cirigliano, M. Ferrario, L. Marazzi, and P. Martelli, "Evidence of Raman-induced polarization pulling," Opt. Express 17, 947 (2009).

[2] V.V. Kozlov, J. Nuno, J. D. Ania-Castanon, and S. Wabnitz, “Theory of fiber optic Raman polarizers," Opt. Lett. 35, 3970-3972 (2010). 